\title{
Household transmission of haemolytic uraemic syndrome associated with Escherichia coli O104:H4 in the Netherlands, May 2011
}

EJ Kuijper (e.j.kuijper@lumc.nl) ${ }^{1}$, D Soonawala ${ }^{2}$, C Vermont $^{3}$, J T van Dissel ${ }^{4}$

1. Department Medical Microbiology, Centre for Infectious Diseases, Leiden University Medical Centre, Leiden, the Netherlands

2. Department of Nefrology, Leiden University Medical Centre, Leiden, the Netherlands

3. Department of Paediatrics, Leiden University Medical Centre, Leiden, the Netherlands

4. Department of Infectious Disease, Centre for Infectious Diseases, Leiden University Medical Centre, Leiden, the Netherlands

Citation style for this article:

Kuijper EJ, Soonawala D, Vermont C, van Dissel JT. Household transmission of haemolytic uraemic syndrome associated with Escherichia coli $\mathrm{O}_{104}$ : $\mathrm{H}_{4}$ in the

Netherlands, May 2011.

Euro Surveill. 2011;16(25):pii=19897. Available online: http://www.eurosurveillance.org/ViewArticle.aspx?Articleld=19897

Article published on 23 June 2011

Following the outbreak of haemolytic uraemic syndrome (HUS) and haemorrhagic colitis in Germany, two patients returning from a stay in Germany developed HUS due to Escherichia coli $\mathrm{O}_{104}: \mathrm{H}_{4}$ in the Netherlands. The index case developed symptoms eight days, and her child 15 days after their return. It is very likely that transmission resulted from secondary spread from mother to child. Recommendations should be made to prevent secondary transmission within households.

\section{Introduction}

Since early May 2011 one of the largest ever reported outbreaks of haemolytic uraemic syndrome (HUS) and bloody diarrhoea caused by Shiga toxin/verotoxinproducing Escherichia coli (STEC/VTEC), also referred to as enterohaemorrhagic $E$. coli (EHEC) has affected Germany [1]. The outbreak strain was identified as an enteroaggregative Shiga toxin-producing $E$. coli 0104: $\mathrm{H}_{4}$ (EAggEC) [2]. Initial findings suggested raw vegetables and salads as vehicle of infections and recommendations were made to abstain from these products. Epidemiological investigations implicated an organic sprout farm in Lower Saxony near Hamburg as the potential source of the outbreak [3]. On 10 June 2011, German public health and food safety authorities issued a joint statement recommending people to abstain from consuming sprouts. A decrease in the number of new cases was seen after 6 June [4].

We describe here two cases of HUS and hemorrhagic colitis that occurred in the Netherlands and were associated with the outbreak in Germany.

\section{Case descriptions}

\section{Patient A}

On 24 May, a woman in her 30 s was admitted to a hospital in the Netherlands with bloody diarrhoea and abdominal pain of two days. Common bacterial gastroenteritis was considered and no antibiotic treatment was initiated. Four days after admission, blood examination revealed severe HUS. The patient was transferred to the Leiden University Medical Centre (LUMC) for haemodialysis which eventually proved not to be necessary.

A tentative diagnosis of HUS due to Stx1-negative, Stx-2 positive, extended spectrum beta-lactamase (ESBL)-producing $E$. coli was confirmed by culture on sorbitol MacConkey agar of a stool sample collected two days after admission to the first hospital. Further identification of $E$. coli serotype 0104 was performed at the Dutch National Institute for Public Health and the Environment (RIVM). A real-time PCR for Stx 2 directly in stool sample was also positive [5]. Stool samples on admission to LUMC were already negative in culture and real-time PCR. Since 30 May 2011, the patient has gradually been recovering from HUS.

Patient A had travelled to Northern Germany for two days in May 2011, accompanied by six relatives, among them her 10 month-old child.

\section{Patient B}

The 10-month-old child of Patient A was examined first on 29 May 2011, one day after transfer of the mother to the LUMC. At the time, the child had mild diarrhoea free of blood, and blood examination did not reveal any abnormality. A stool sample was collected and tested positive for Stx2 in the real-time PCR. Culture revealed growth of ESBL-positive E. coli 0104. On 30 May, the child developed fever and blood abnormalities compatible with HUS, and was admitted to hospital. The next day, it developed neurological symptoms, i.e. seizures, and was transferred to a specialised child dialysis centre, where experimental treatment with eculizumab was started. The patient received prolonged mechanical ventilation and inotropic therapy. Three weeks after transfer, the patient is still on dialysis and the neurological outcome is unsure. 
Family members

Of the other five relatives of Patients $A$ and $B$ who had also travelled to Northern Germany, one developed mild diarrhoea 16 days after their return, but microbiological examinations were not performed since the diarrhoea resolved one day later and no HUS developed. The remaining relatives did not develop any symptoms and were not investigated for the presence of $E$. coli 0104 .

\section{Microbiological and public health implications}

HUS is a rare disease in the Netherlands, with approximately 20 patients per year [6]. During the outbreak in Germany, 854 cases of HUS and 2,848 non-HUS STEC/VTEC cases have been reported as of 22 June, of which $4.6 \%$ and $2.2 \%$, respectively, were found in other Member States of the European Union [7]. In the Netherlands, four HUS (including Patients $A$ and $B$ described here) and five non-HUS laboratory-confirmed STEC/VTEC cases were detected. All acquired the infection during a recent visit to Germany.

Microbiological examination of stool samples from Patient $A$ gave negative results in the real-time PCR and culture at the time of transfer to another hospital when HUS had fully developed, six days after disease onset, indicating the need to apply diagnostic tests early in the disease. Similarly, Patient B had positive faeces tests and excreted the organism before haemorrhagic colitis and HUS developed.

We hypothesise that Patient B probably acquired HUS by secondary transmission within the household, because the child developed illness only 15 days after return form Northern Germany. The child had spent much time with her mother during her stay at the first hospital, when the mother developed diarrhoea. Before admission of the child to LUMC, the mother had not been advised to take any specific hygienic measures. The child was not breastfed at the time. The age of the child makes it unlikely that it had eaten a food product such as sprouts.

Recently, a group of investigators reported that the strain causing the outbreak in Germany is in fact not a typical virulent Shiga toxin-producing Escherichia coli strain, but instead is a more rare, hybrid pathotype that harbours the phage encoded the Shiga toxin gene in an EAggEC background [2]. These findings are relevant for understanding the epidemiology since EAggEC is a common pathogen causing diarrhoea in travellers and persistent diarrhoea in infants and young children living in countries with poor sanitation. In contrast to STEC/VTEC strains, which that have an animal reservoir, mostly ruminants, EAggEC strains probably have a human reservoir only.

Secondary transmission frequently occurs in outbreaks caused by classical EHEC 0157. A review of 90 confirmed outbreaks in the United Kingdom, Ireland,
Scandinavia, Canada, the United States and Japan revealed that approximately $20 \%$ of all outbreak cases were the result of secondary spread [8]. Interestingly, the spread was significantly influenced by age and modes of transmission. A lower median age of the index patients was associated with a higher rate of secondary cases and household contacts aged one to four years were most likely to become infected. Immediate separation of a paediatric patient from its siblings when there is a clinical suspicion of STEC/ VTEC $0_{157}$ infection has been suggested as an important measure in the prevention of secondary cases [9]. Isolation of all symptomatic primary patients immediately after they receive a microbiological diagnosis of STEC/VTEC 0157 infection could potentially decrease the number of secondary household cases by $50 \%$ [9]. In this family however, the primary case patient was an adult. Since young children usually have extensive close contacts with their parents; separation of young children from a parent with a suspected STEC/VTEC or E. coli $\mathrm{O}_{104}$ infection should be considered in order to prevent secondary transmission to the child.

\section{Acknowledgements:}

We thank dr. E. Franz (Laboratory for Zoonoses and Environmental Microbiology, Centre for Infectious Disease Control Netherlands, National Institute for Public Health and the Environment), dr. A Timen (Centre for Infectious Disease Control, the Netherlands), dr. E. Wessels (Leiden University Medical Centre) and dr. P. Voorn (St. Antonius Hospital, Nieuwegein, the Netherlands) for their contribution for this short report.

\section{References}

1. Frank C, Faber MS, Askar M, Bernard H, Fruth A, Gilsdorf $A$, et al. Large and ongoing outbreak of haemolytic uraemic syndrome, Germany, May 2011. Euro Surveill. 2011;16(21):pii=19878. Available from: http://www. eurosurveillance.org/ViewArticle.aspx?Articleld=19878

2. Scheutz F, Møller Nielsen E, Frimodt-Møller J, Boisen $\mathrm{N}$, Morabito S, Tozzoli R, et al. Characteristics of the enteroaggregative Shiga toxin/verotoxin-producing Escherichia coli $0104: \mathrm{H}_{4}$ strain causing the outbreak of haemolytic uraemic syndrome in Germany, May to June 2011. Euro Surveill. 2011;16(24):pii=19889. Available from: http://www. eurosurveillance.org/ViewArticle.aspx?Articleld=19889

3. Joint statement issued by the Federal Institute for Risk Assessment (BfR), Federal Office of Consumer Protection and Food safety (BVL), Robert Koch Institute (RKI). Press release. Berlin: BfR, Braunschweig: BVL, Berlin: RKI; 10 June 2011. Available from: http://ecdc.europa.eu/en/press/news/ Documents/1106_Joint_Press_Release_German_authorities_ issue_a_joint_statement.pdf

4. Informationen zum EHEC/HUS-Ausbruchsgeschehen [Information on the EHEC/HUS outbreak events]. Data from 21 Jun 2011, 10 pm. Berlin: Robert Koch Institut; 22 Jun 2011 11 am. German. Available from: http://www.rki.de/cln_16o/ nn_205760/DE/Home/Info-HUS.html

5. Schuurman T, Roovers A, van der Zwaluw WK, van Zwet AA, Sabbe LJ, Kooistra-Smid AM, et al. Evaluation of 5'-nuclease and hybridization probe assays for the detection of shiga toxin-producing Escherichia coli in human stools. J Microbiol Methods. 2007;70(3):406-15.

6. Havelaar AH, Van Duynhoven YT, Nauta MJ, Bouwknegt $M$, Heuvelink AE, De Wit GA, et al. Disease burden in The Netherlands due to infections with Shiga toxin-producing Escherichia coli 0157. Epidemiol Infect. 2004;132(3):467-84. 
7. European Centre for Disease Prevention and Control (ECDC). Outbreak of Shiga toxin-producing E. coli in Germany (22 June 2011, 11:00). Stockholm: ECDC; 22 Jun 2011. Available from: http://www.ecdc.europa.eu/en/activities/sciadvice/Lists/ ECDC\%20Reviews/ECDC_DispForm.aspx?List $=512 \mathrm{ff} 74 \mathrm{f}-77 \mathrm{~d} 4$ 4ad8-b6d6-bfof $23083 f 30 \& I D=1119$ \&RootFolder $=\% 2$ Fen $\% 2$ Fact ivities\%2Fsciadvice \%2FLists\%2FECDC\%20Reviews

8. Snedeker KG, Shaw DJ, Locking ME, Prescott R. Primary and secondary cases in Escherichia coli 0157 outbreaks: a statistical analysis. BMC Infect Dis. 2009;9:144.

9. Werber D, Mason BW, Evans MR, Salmon RL. Preventing Household Transmission of Shiga Toxin-Producing Escherichia coli 0157 Infection: Promptly Separating Siblings Might Be the Key. Clin Infect Dis. 2008;46(8):1189-96. 